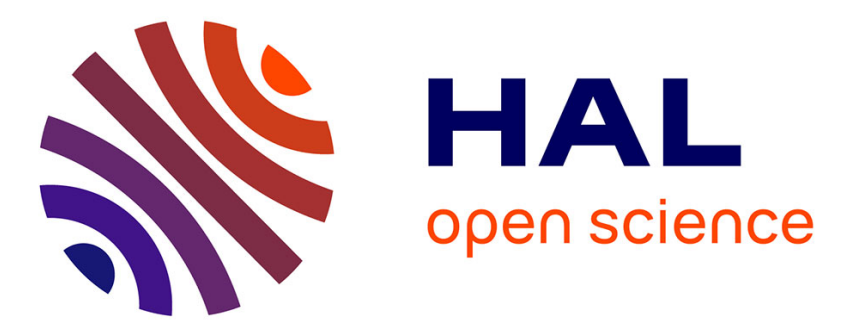

\title{
Shape-Constrained Level Set Segmentation for Hybrid CPU-GPU Computers
}

\author{
Souleymane Balla-Arabé, Xinbo Gao, Dominique Ginhac, Fan Yang
}

\section{To cite this version:}

Souleymane Balla-Arabé, Xinbo Gao, Dominique Ginhac, Fan Yang. Shape-Constrained Level Set Segmentation for Hybrid CPU-GPU Computers. Neurocomputing, 2016, 177, pp.40-48. 10.1016/j.neucom.2015.11.004 . hal-01430277

\section{HAL Id: hal-01430277 \\ https://u-bourgogne.hal.science/hal-01430277}

Submitted on 9 Jan 2017

HAL is a multi-disciplinary open access archive for the deposit and dissemination of scientific research documents, whether they are published or not. The documents may come from teaching and research institutions in France or abroad, or from public or private research centers.
L'archive ouverte pluridisciplinaire HAL, est destinée au dépôt et à la diffusion de documents scientifiques de niveau recherche, publiés ou non, émanant des établissements d'enseignement et de recherche français ou étrangers, des laboratoires publics ou privés. 


\title{
Shape-Constrained Level Set Segmentation for Hybrid CPU-GPU Computers
}

\author{
Souleymane Balla-Arabé,Xinbo Gao ,Dominique Ginhac, Fan Yang
}

\begin{abstract}
Due to its intrinsic advantages such as the ability to handle complex shapes, the level set method (LSM) has been widely applied to image segmentation. Nevertheless, the LSM is computationally expensive. In order to improve the performance of the traditional LSM both in terms of efficiency and effectiveness, we propose a novel algorithm based on the lattice Boltzmann method (LBM). Using local region statistics and prior shape, we design an effective and local speed function for the LSM, from which we deduce a shape prior based body force for LBM solver. An NVIDIA graphics processing units (GPU) is used to accelerate the method. Ourintroduced algorithm has several advantages. First, it is accurate even if they are some geometric transformations (rotation angle, scaling factor and translation vector) betweenthe object to be segmented and the prior shape. Second, it is local and therefore suitable for massively parallel architectures. Third, the use of local region information allows it to deal with intensity inhomogeneities. Fourth, including shape prior allows the method to handle occlusion and noise. Fourth, the model is fast. Finally the algorithm can be used without shape prior by means of minor modification. Intensive Experiments demonstrate, objectively and subjectively, the performance of the introduced framework.
\end{abstract}

2015 Elsevier Science B.V. All rights reserved

Keywords:image segmentation,Massively parallel architectures, Partial differential equations, Shape prior, level set method.

\section{Corresponding author}

Xinbo Gao(M'02-SM'07) received the B.Eng., M.Sc. and Ph.D. degrees in signal and information processing from Xidian University, China, in 1994, 1997 and 1999 respectively. From 1997 to 1998, he was a research fellow in the Department of Computer Science at Shizuoka University, Japan. From 2000 to 2001, he was a postdoctoral research fellow in the Department of Information Engineering at the Chinese University of Hong Kong. Since 2001, he joined the School of Electronic Engineering at Xidian University. Currently, he is a Professor of Pattern Recognition and Intelligent System, Director of the VIPS Lab, and the Assistant of the President of Xidian University. His research interests are computational intelligence, machine learning, computer vision, pattern recognition and wireless communications. In these areas, he has published 5 books and around 150 technical articles in refereed journals and proceedings including IEEE TRANSACTIONS ON IMAGE PROCESSING, the IEEE TRANSACTIONS ON CIRCUITS AND SYSTEMS FOR VIDEO TECHNOLOGY, the IEEE TRANSACTIONS ON NEURAL NETWORKS, and the IEEE TRANSACTIONS ON SYSTEMS, MAN, AND CYBERNETICS. He is on the editorial boards of several journals including Signal Processing (Elsevier), and Neurocomputing (Elsevier). He served as general chair/co-chair or program committee chair/co-chair or PC member for around 30 major international conferences. Now, he is a Fellow of IET and Senior Member of IEEE.

E-Mail: xbgao.xidian@gmail.com

Phone: +86-29-88201838

Fax: $+86-29-88201620$

Homepage: http://see.xidian.edu.cn/faculty/xbgao/ 


\title{
Shape-Constrained Level Set Segmentation for Hybrid CPU-GPU Computers
}

\author{
Souleymane Balla-Arabé,Xinbo Gao ,Dominique Ginhac, Fan Yang
}

\begin{abstract}
Due to its intrinsic advantages such as the ability to handle complex shapes, the level set method (LSM) has been widely applied to image segmentation. Nevertheless, the LSM is computationally expensive. In order to improve the performance of the traditional LSM both in terms of efficiency and effectiveness, we propose a novel algorithm based on the lattice Boltzmann method (LBM). Using local region statistics and prior shape, we design an effective and local speed function for the LSM, from which we deduce a shape prior based body force for LBM solver. An NVIDIA graphics processing units (GPU) is used to accelerate the method. Our introduced algorithm has several advantages. First, it is accurate even if they are some geometric transformations (rotation angle, scaling factor and translation vector) betweenthe object to be segmented and the prior shape. Second, it is local and therefore suitable for massively parallel architectures. Third, the use of local region information allows it to deal with intensity inhomogeneities. Fourth, including shape prior allows the method to handle occlusion and noise. Fourth, the model is fast. Finally the algorithm can be used without shape prior by means of minor modification. Intensive Experiments demonstrate, objectively and subjectively, the performance of the introduced framework.
\end{abstract}

2015 Elsevier Science B.V. All rights reserved

Keywords: image segmentation,Massively parallel architectures, Partial differential equations, Shape prior, level set method.

\section{Introduction}

In computer vision and pattern recognition, image segmentation[1]-[2], [32]-[35] is a major process by which a given image is partitioned into number of meaningful and homogeneous regions, such that the union of any two neighborhood regions yields a heterogeneous segment. The task is non-trivial and more challenging in presence of noise, intensity inhomogeneities or occlusion.

Recently, optimization methods have been widely used as highly effective image segmentation strategies. They basically achieve the segmentation by minimizing a given energy function designed from the image information. Optimization methods can be roughly classified into two important classes: spatially discrete and spatially continuous representations. In spatially discrete approaches, the pixels of the image are usually considered as the nodes of a graph, and the aim of segmentation is to find cuts of this graph which have a minimal cost[25]-[26].

As shown by Fig. 1, Active contour models (ACMs) belong to the spatially continuous approaches: the segmentation of the image plane is considered as a problem of infinite-dimensional optimization.In this class, variational methods are used in the image segmentation. The principle is to evolve the active curve in the direction of the negative energy gradient by means of an appropriate partial differential equation. The implicit active curve method or level set method (LSM)[3]-[7] designates the class of ACM which uses the Eulerian framework, that is, the geometric representation of the evolving curve, instead of the parametric one, that is, the Lagrangian framework[27]-[29].

\footnotetext{
" Corresponding author: Tel.: +862988201838; fax: +862988201620. E-mail address: xbgao@ mail.xidian.edu.cn
} 
The LSM has several intrinsic advantages, such as the ability to easily handle complex shapes, and topological changes comparable to parametric active contours. It is, furthermore, straightforward to pass from two-dimensional (2D) to 3D space, and to add some constraints on the smoothness of the boundaries via some regularization terms.

The original idea of the LSM stems from the Hamilton Jacobiapproach, i.e., a time-dependent equation for a moving surface[8]-[9]. In two-dimensional (2D) space, the LSM aims to evolve a given curve toward its interior or exterior normal until defining the boundary of the object of interest. The curve evolution is driven by the level set equation (LSE) which is a partial differential equation and, in its simple form can be expressed as

$$
\frac{\partial \phi}{\partial t}=V|\nabla \phi|
$$

where $\phi$ is the level set function (LSF), $\nabla \phi$ is the gradient of $\phi$ and $V$ is the speed function which drives the active contour towards the region boundaries. Two approaches are usually used to stop the evolving curve on the boundary of the desired object.The first one uses an edge indicator depending on the gradient of the image like in classical snakes and active contour models[10]-[12]. The second one uses some regional attributes to stop the evolving curve on the actual boundary[13]-[14]. The latter is more robust against noise and can detect objects with weak edges. One of the most interesting approaches was proposed in[15] where Chan and Vese introduced a level set formulation to minimize the Mumford and Shah functional[16]. They converted the problem into a mean curvature flow problem just like the active contours. But the results were better than the classical active contours because the stopping term did not depend on the gradient of the image which reduces the dependence on strong edges and improves the robustness against noise. However the method cannot deal with intensity inhomogeneities, and is not suitable for parallel programming because at each iteration the average intensities inside and outside the contour should be computed, which increases drastically the CPU time by increasing communications between processors. Furthermore, in order to solve the LSE, most of the available methods suggest the use of some computational expensive finite difference, finite element or finite volume approximations and an explicit computation of the curvature[17].

In[18], the authors used the lattice Boltzmann method (LBM) as an alternative approach for solving the LSE for region-based image segmentation. The method can better handle the problem of time consuming because the curvature is implicitly computed and the algorithm is simple. Nevertheless, in this method the proposed stop function is not local, thus cannot deal with intensity inhomogeneities and at the same time is not suitable for GPU-based computations.

In the present paper, we propose a new supervised method which improves and overcomes some limitations of the one proposed in [18]. Using local region properties of the image, we design a new localized stop function which includes shape prior information. The prior shape is used to roughly guide the evolving curve toward boundaries of the object to detect, and the local region statistics allow the detection of fine details. Moreover, since sometimes they are some small geometric transformations (rotation angle, scaling factor and translation vector) between the object to be segmented and the prior shape, we introduced a straightforward adjustment method which effectively increases the accuracy of the method. The proposed algorithm can deal with intensity inhomogeneities, occlusion and noise, and is highly parallelizable due to its local property. Furthermore, it can be easily used without shape prior by means of minor modification. 


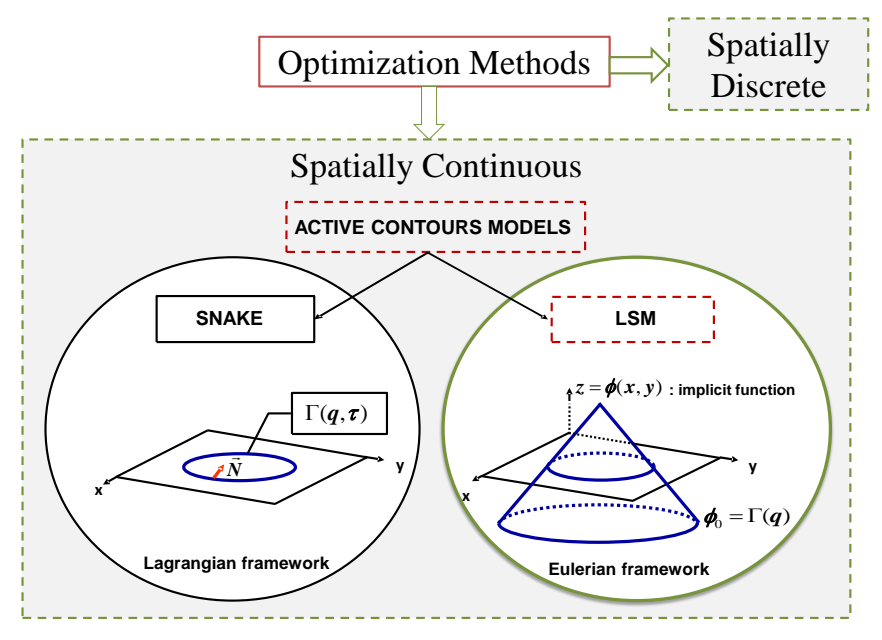

Fig. 1.Organization of optimization methods.

The rest of this paper is organized as follows: Section II briefly introduces the LBM. In section III,we present the formulation of the proposed method. Section IV validates the performance of the proposed image segmentation method through experimental results. Section V concludes the paper.

\section{Background}

This section gives an overview of the LBM method which is used in this paper to solve the LSE.The LBM models Boltzmann particle dynamics on a 2D or 3D lattice. At first, it was designed to solve macroscopic fluid dynamics problems. The LBM is second-order accurate both in time and space, and in the limit of zero time step and lattice spacing, it yields the Navier-Stokes equations for an incompressible fluid [19].

In this paper, we use the D2Q9 (2D with 8 links with its neighbors and one link for the cell itself) LBM lattice structure. Fig. 2 shows a typical D2Q9 model.

The evolution equation of LBM can be written as

$$
f_{i}\left(\vec{r}+\vec{e}_{i}, t+1\right)=f_{i}(\vec{r}, t)+\frac{1}{\tau}\left[f_{i}^{e q}(\vec{r}, t)-f_{i}(\vec{r}, t)\right]+\frac{D}{b c^{2}} \cdot \vec{F} \cdot \vec{e}_{i},(2)
$$

where $\vec{e}_{i}$ is the velocity vector of a given link $i, f_{i}(\vec{r}, t)$ the distribution of the particle that moves along that link, $t$ the time, $\vec{r}$ the position of the cell, $\vec{F}$ the body force, $D$ the grid dimension, $b$ the link at each grid point and $c$ the length of each link which is set to 1 in this paper. The parameter $\tau$ represents the relaxation time determining the kinematic viscosity $\vartheta$ of the fluid by

$$
\vartheta=\frac{1}{3}\left(\tau-\frac{1}{2}\right)
$$

and $f_{i}^{e q}$ is the local equilibrium particle distribution usually given by the Bhatnager, Gross, Krook (BGK) model

$$
f_{i}^{e q}(\rho, \vec{u})=\rho\left(A_{i}+B_{i}\left(\vec{e}_{i} \cdot \vec{u}\right)+C_{i}\left(\vec{e}_{i} \cdot \vec{u}\right)^{2}+D_{i}(\vec{u})^{2}\right),
$$




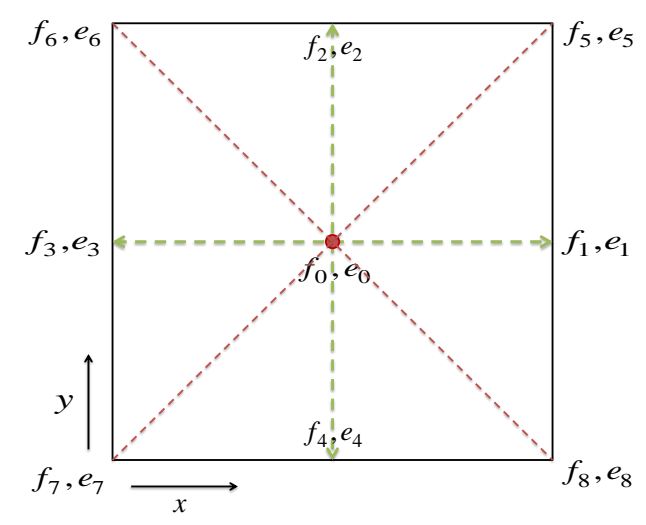

Fig. 2. Spatial structure of the D2Q9 LBM lattice.

where the constant coefficients $A_{i}$ to $D_{i}$ are chosen via the geometry of the lattice links, $\rho$ and $\vec{u}$ are respectively the macroscopic fluid density and velocity computed from the particle distributions as

$$
\begin{aligned}
& \rho=\sum_{i} f_{i}, \\
& \vec{u}=\frac{1}{\rho} \sum_{i} f_{i} \vec{e}_{i} .
\end{aligned}
$$

For diffusion problems, the equilibrium function can be simplified as [20]

$$
f_{i}^{e q}(\rho, \vec{u})=\rho A_{i} .
$$

In the case of D2Q9 model, $A_{i}=4 / 9$ for the zero link, $A_{i}=1 / 9$ for the axial links, $A_{i}=1 / 36$ for the diagonal links and the relaxation time $\tau$ is then determined using the following equation

$$
\xi=\frac{2}{9}(2 \tau-1)
$$

where $\xi$ is the diffusion coefficient. By performing the Chapman-Enskog analysis the following diffusion equation can be recovered from the LBM evolution equation (Eq. (2)),

$$
\frac{\partial \rho}{\partial t}=\xi \operatorname{div}(\nabla \rho)+F
$$

where div is the divergence operator. Substituting $\rho$ by the signed distance function $\phi$ in Eq. (9), the LSE can be recovered. The body force $F$ represents the link with the image data in the LBM solver.

Another approach for the LBM based level set segmentation is to add a medium between the nodes of the lattice [21] in order to design a stop function for the evolving active contour. The particles can pass through the medium with a possibility of $g_{i}(\vec{r})$, and will be punched back where they were with a possibility of $1-g_{i}(\vec{r})$. They therefore modified the LBM evolution equation as 


$$
\begin{aligned}
f_{i}\left(\vec{r}+\vec{e}_{i}, t+1\right)= & g_{i}(\vec{r})\left[f_{i}(\vec{r}, t)+\frac{1}{\tau}\left[f_{i}^{e q}(\vec{r}, t)-f_{i}(\vec{r}, t)\right]+\sigma\right], \\
& +\left(1-g_{i}(\vec{r})\right) f_{i}\left(\vec{r}+\vec{e}_{i}, t\right)
\end{aligned}
$$

where $\sigma$ is the convection coefficient.

\section{TheShape-Constrained Level Set Algorithm}

In this section, we first design and analyze the proposed localized shape prior algorithm, and then we give the main implementation steps.

3.1 Design and analysis of the proposed algorithm

For the level set equation defined in (Eq. (1)), we design the following shape prior based speed function

$$
\mathrm{V}(\vec{r})=\alpha\left(f_{\text {shape }}(\vec{r})-\frac{e_{1}(\vec{r})+e_{2}(\vec{r})}{2}\right)+\xi(\vec{r}),
$$

with

$$
f_{\text {shape }}(\vec{r})=I(\vec{r})+\lambda\left(\operatorname{sign}\left(\phi_{\text {ref }}(\vec{r})\right)-\operatorname{sign}(\phi(\vec{r}))\right),
$$

where $\vec{r}$ is a spatial variable, $\alpha$ a controlling parameter, $\lambda$ a weighting positive parameter, $\phi$ the level set function which is a signed distance function, $\phi_{\text {ref }}$ a signed function obtain from the aligned prior shape, and $I$ the pixel intensity. In this paper, $\phi$ is positive inside the contour and negative outside, just as $\phi_{r e f}$ is positive inside the aligned prior shape and negative outside.

The terms $e_{1}$ and $e_{2}$ are local mean values of a given pixel respectively inside and outside the evolving curve. They are defined as

$$
\begin{gathered}
e_{1}(\vec{r})=\frac{\int_{\Omega} K(\vec{r}-\vec{s}) \cdot I(\vec{s}) \cdot H(\phi) d \vec{s}}{\int_{\Omega} K(\vec{r}-\vec{s}) \cdot H(\phi) d \vec{s}}, \\
e_{2}(\vec{r})=\frac{\int_{\Omega} K(\vec{r}-\vec{s}) \cdot I(\vec{s}) \cdot(1-H(\phi)) d \vec{s}}{\int_{\Omega} K(\vec{r}-\vec{s}) \cdot(1-H(\phi)) d \vec{s}},
\end{gathered}
$$

where $H$ is the Heaviside function and

$$
K(\vec{r}-\vec{s})=\left\{\begin{array}{l}
1,\|\vec{r}-\vec{s}\|<\mu \\
0, \text { otherwise }
\end{array}\right.
$$

with $\mu$ the radius parameter and $\vec{s}$ a spatial variable. $K$ is a characteristic function used to mask local regions, it will be 1 when the point $\vec{s}$ is within a ball of radius $\mu$ centered at $\vec{r}$, and 0 otherwise.

The term $\xi(\vec{r})$ is the non-linear curvature term used to smooth the contour. It can be written as 


$$
\xi(\vec{r})=\beta \nabla \cdot \frac{\nabla \phi}{|\nabla \phi|},
$$

where $\beta$ is a controlling parameter.

The proposed shape prior based level set equation is therefore

$$
\begin{aligned}
\frac{\partial \phi}{\partial t} & =\alpha\left(I(\vec{r})+\lambda\left(\operatorname{sign}\left(\phi_{r e f}(\vec{r})\right)-\operatorname{sign}(\phi(\vec{r}))\right)\right. \\
& \left.\left.-\frac{e_{1}(\vec{r})+e_{2}(\vec{r})}{2}\right)+\beta \nabla \cdot \frac{\nabla \phi}{|\nabla \phi|}\right)|\nabla \phi| .
\end{aligned}
$$

In order to make the method suitable for parallel programming, we use the local LBM to solve the proposed LSE. Since we consider $\phi$ as a signed distance function, i.e., $|\phi|=1$, Eq. (17) can therefore be expressed as

$$
\begin{aligned}
\frac{\partial \phi}{\partial t} & =\alpha\left(I(\vec{r})+\lambda\left(\operatorname{sign}\left(\phi_{r e f}(\vec{r})\right)-\operatorname{sign}(\phi(\vec{r}))\right)\right. \\
& \left.-\frac{e_{1}(\vec{r})+e_{2}(\vec{r})}{2}\right)+\beta \nabla \cdot \nabla \phi,
\end{aligned}
$$

which is similar to Eq. (9) with the body force defined as

$$
\begin{aligned}
F(\vec{r}) & =\alpha\left(I(\vec{r})+\lambda\left(\operatorname{sign}\left(\phi_{\text {ref }}(\vec{r})\right)-\operatorname{sign}(\phi(\vec{r}))\right)\right. \\
& \left.-\frac{e_{1}(\vec{r})+e_{2}(\vec{r})}{2}\right) .
\end{aligned}
$$

The proposed level set equation can thus be solved using the lattice Boltzmann evolution equation Eq. (2) without the necessity of explicitly computes the computational expensive curvature term since it is implicitly handled.Furthermore, since the above defined body force is also local, it will not affect the local nature of the LBM. This will result in an adequate algorithm to massively parallel devices such as the GPU because the time consuming communications between the processors will be greatly reduced, as well as the executive time.

From Eqs. (11) and (12) we can do the followings remarks:

1- Considering an object with or without edges, $V(\vec{r})$ reduces to zero when the active pixel intensity verify

$$
I(\vec{r}) \approx \frac{e_{1}(\vec{r})+e_{2}(\vec{r})}{2}-\lambda\left(\operatorname{sign}\left(\phi_{r e f}(\vec{r})\right)-\operatorname{sign}(\phi(\vec{r}))\right),
$$

2- In the case of partial occlusion, the second term in the right hand of Eq. (20) makes the contour continue to evolve instead to stop on the false boundaries caused by the occlusion.

3- If the image template is not available, we just have to fix $\phi_{r e f}=\phi$ or $\lambda=0$ in the algorithm and to perform the segmentation without prior shape.

According to the above statements, using $V(\vec{r})$ as a stop function will theoretically produce interesting results. This will be confirmed by experimental results.

For the shape adjustment, we use the following method which can handle the cases of geometric transformations (translation, rotation and scaling). Let consider Fig. 3 where $\theta$ is the rotation angle. 
In order to determine $\theta$, the translation parameter $\vec{H}$ and the scaling parameter $S$, we have the following steps. We first choose two remarkable points on the shape prior ( $A$ and $B$ ), which can be easily recover on the object to be segmented $(C$ and $D)$. In this paper, the selected points are vertex of some well-defined angles. $O_{1}$ and $O_{2}$ are respectively the centers of the $[A B]$ and $[C D]$. The rotation angle is therefore the angle between the straight lines $(A B)$ and $(C D)$. By considering the scalar product of the vectors $\overrightarrow{A B}$ and $\overrightarrow{C D}$, we have

$$
\begin{aligned}
\overrightarrow{A B} \cdot \overrightarrow{C D} & =|\overrightarrow{A B}| \times|\overrightarrow{C D}| \times \cos (\theta) \\
& =\left(x_{B}-x_{A}\right)\left(x_{D}-x_{C}\right)+\left(y_{B}-y_{A}\right)\left(y_{D}-y_{C}\right) .
\end{aligned}
$$

We can then compute the rotation angle $\theta$ since

$$
\cos (\theta)=\frac{1}{S} \cdot \frac{\left(x_{B}-x_{A}\right)\left(x_{D}-x_{C}\right)+\left(y_{B}-y_{A}\right)\left(y_{D}-y_{C}\right)}{\left(x_{B}-x_{A}\right)^{2}+\left(y_{B}-y_{A}\right)^{2}},
$$

where $S$ is the scaling parameter defined as

$$
\begin{aligned}
S & =|\overrightarrow{C D}| /|\overrightarrow{A B}| \\
& =\sqrt{\left[\left(x_{D}-x_{C}\right)^{2}+\left(y_{D}-y_{C}\right)^{2}\right] /\left[\left(x_{B}-x_{A}\right)^{2}+\left(y_{B}-y_{A}\right)^{2}\right]} .
\end{aligned}
$$

In the shape adjustment process, after the scaling and the rotation steps, the translation parameter $\vec{H}$ can be written as

$$
\vec{H}=\vec{H}_{0}=\left\{\begin{array}{l}
\frac{1}{2}\left(x_{C}+x_{D}\right)-\frac{1}{2}\left(x_{A}+x_{B}\right) \\
\frac{1}{2}\left(y_{C}+y_{D}\right)-\frac{1}{2}\left(y_{A}+y_{B}\right)
\end{array} .\right.
$$

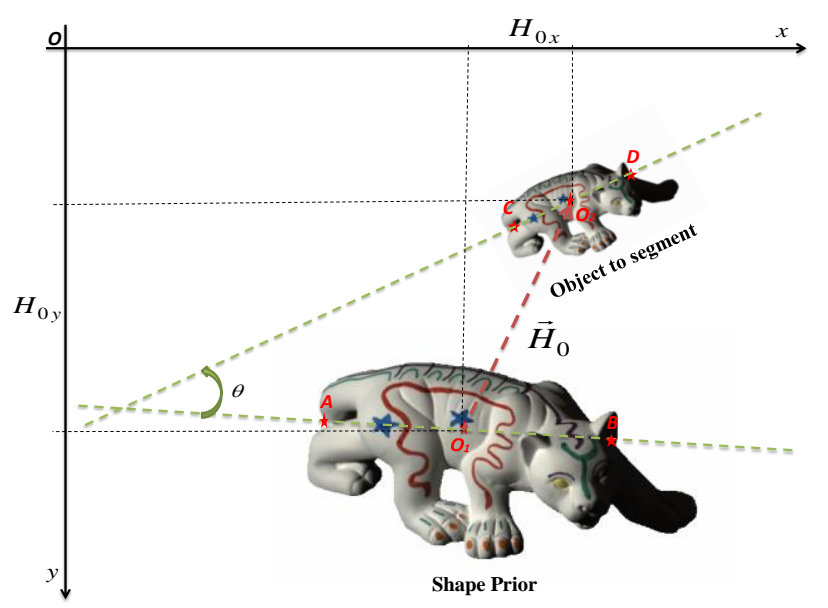

Fig. 3.Shape adjustment parameters.

Note that before the scaling and the rotation steps in the shapealignment process, we have $\vec{H} \neq \vec{H}_{0}$. 


\subsection{Implementation}

The steps for the implementation of the proposed algorithm are outlined as follows:

a) Initialize $\phi$ as a signed distance function;

b) Do the shape alignment, which consists to first put the template at the same scale with the object to segment, the scaling parameter being $S$. The second step is the rotation according to $\theta$, and the last step consists to translate the geometric template according to $\vec{H}$;

c) Compute $e_{1}$ and $e_{2}$ according to Eqs. (13) and (14);

d) Calculate $F(\vec{r})$ according to Eq. (19);

e) Resolve the proposed level set equation with the LBM evolution equation Eq. (2);

f) Accumulate the $f_{i}(\vec{r}, t)$ values at each grid point using Eq. (5) to get the updated values of $\phi$ at each point;

g) Find the contour which is the zero level of $\phi$;

h) If the algorithm has not converged, i.e., $\left\|\phi^{t+1}-\phi^{t}\right\|>10^{-5}$, go back to step c).

Fig. 4 illustrates the flowchart of the introduced supervised level set based algorithm. It can be seen that the proposed method is simple to implement since the LBM eliminates the use of complex approximations to solve the LSE and the need to explicitly compute the curvature.

\section{Experimental Results Analysis}

In this section we firstly demonstrate the performance of the proposed image segmentation method in term of efficiency and efficacy in presence of intensity inhomogeneities and object with weak or without edges. Four level set based segmentation methods: the Chan and Vese's method (CV)[15], the Li's method[22], the Chen's method[21] and the Aaron and Zhao's method (AZ)[23] are selected as benchmarks. Secondly, we demonstrate the effectiveness of the proposed method in the case of partial occlusion and noise corruption. Finally, we show that the our method is effective even without prior shape.

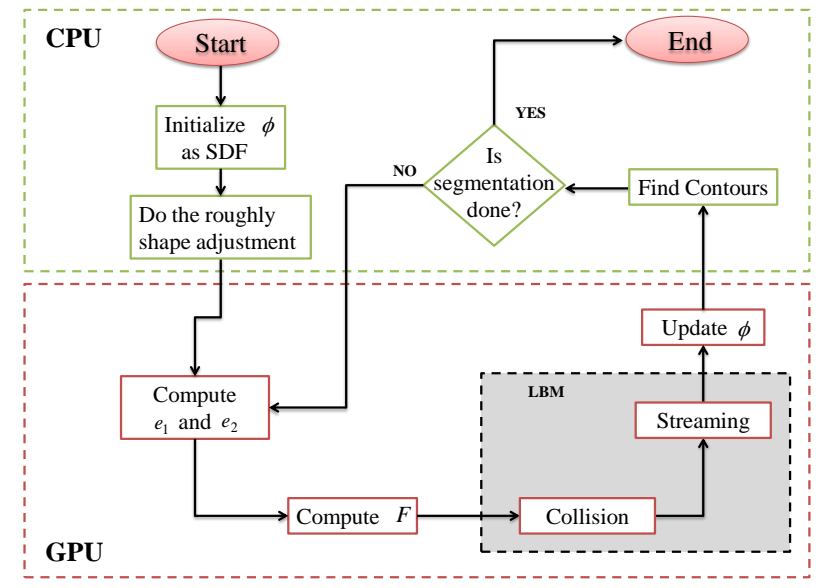

Fig. 4. Flowchart representing the process of the introduced framework. 
The experimental environment is Matlab R2012b installed on a PC AMD Athlon (tm) 5200 processor with a clock speed of $2.31 \mathrm{GHz}$ and $2 \mathrm{~GB}$ of RAM, and possessing the NVIDIA GPU GT 430. The optimized Matlab function arrayfun is used to execute the introduced level set algorithm on the GPU.For example, the body force is computed using the following instructions

$$
\begin{array}{ll}
1- & I d=\operatorname{gpuArray}(I) ; \\
2- & \phi d=\operatorname{gpuArray}(\phi) ; \\
3- & \phi_{\text {ref }} d=\operatorname{gpuArray}\left(\phi_{\text {ref }}\right) ; \\
4- & F d=\operatorname{arrayfun}\left(@ B o d y_{-} \text {force, Id }, \phi d, \phi_{\text {ref }} d\right) .
\end{array}
$$

The first, the second and the third instructions transfer respectively $I, \phi$ and $\phi_{\text {ref }}$ from the CPU to the GPU, while the fourth instruction computes the body force on the GPU using the kernel function Body_force.m programmed according to Eq. (19). Since all the output arguments are stored in the GPU memory, the function gather is used to transfer them back from GPU to CPU.In all the experiments the radius parameter $\mu$ is set to 4 , the weighting parameter $\lambda$ to 3 and the controlling parameter $\alpha$ also to 3 .

The prior shape of the corpus callosum used in the experiments is the mean shape of human's segmentation of a training set. In the case of natural images, the more suitable prior shapes have been determined in a dataset of possible prior shapes. Then, they have been rescaled, translated and/or rotated in order to make the experimental cases more difficult.For more information about prior shapes detection or construction, one can refer to[30]-[31].

To objectively evaluate the performance of the proposed method, we use the Levine and Nazif (LN) inter-region and intra-region contrast criterion[24]. The lower is the intra-region contrast, the better is segmentation result, and the higher is the inter-region contrast, the better is the segmentation result.

Fig. 5 demonstrates the proposed method on an MRI image of head and some real world images. Row I displays the prior shapes, row II the initial contours, row III the results of the Chen's method, row IV the results of the Li's method, row V the results of AZ method, row VI the results of the CV's method and row VII the results of the proposed method. The executive times are displayed in Table 1 and the objective evaluation results in Tables 2 and 3. In nearly all the presented cases the proposed method has the lowest intra-region contrast and the highest inter-region contrast. Thus, it objectively gives the best results, which can be confirmed subjectively by observing the segmented images. The Chen's and the Li's methods fail in most of the cases because they are edge based and the objects are with weak or without edges. The CV's method is based on non-local means values and is therefore not effective when they are intensity inhomogeneities like in most of the presented cases. In column (a), only the proposed method has well segmented the corpus callosum in the head magnetic resonance image. Furthermore it is the fastest one; in this case the executive time is of $0.3465 \mathrm{~s}$ versus $41.89 \mathrm{~s}$ for the CV's method. In column (b) and (c), the results of the proposed method are also pretty good, and demonstrate its effectiveness in presence of intensity inhomogeneities, weak edges and for texture image segmentation.

Fig. 6 shows the performance of the proposed method in the case of partial occlusion. In all the cases, the segmentation results are promising.

Fig. 7 shows the performance of the proposed method in presence of noise. In row II the image is corrupted by the speckle noise with the variance $v=0.04$; in row III the image is corrupted by the Gaussian noise with the mean $m=0.3$ and the variance $v=0.3$; in row IV the image is corrupted by salt and pepper noise with the density $d=0.3$; finally in row $\mathrm{V}$ the image is corrupted by the poisson 
noise. The quality of the segmentation result demonstrates the robustness against noise of our method.
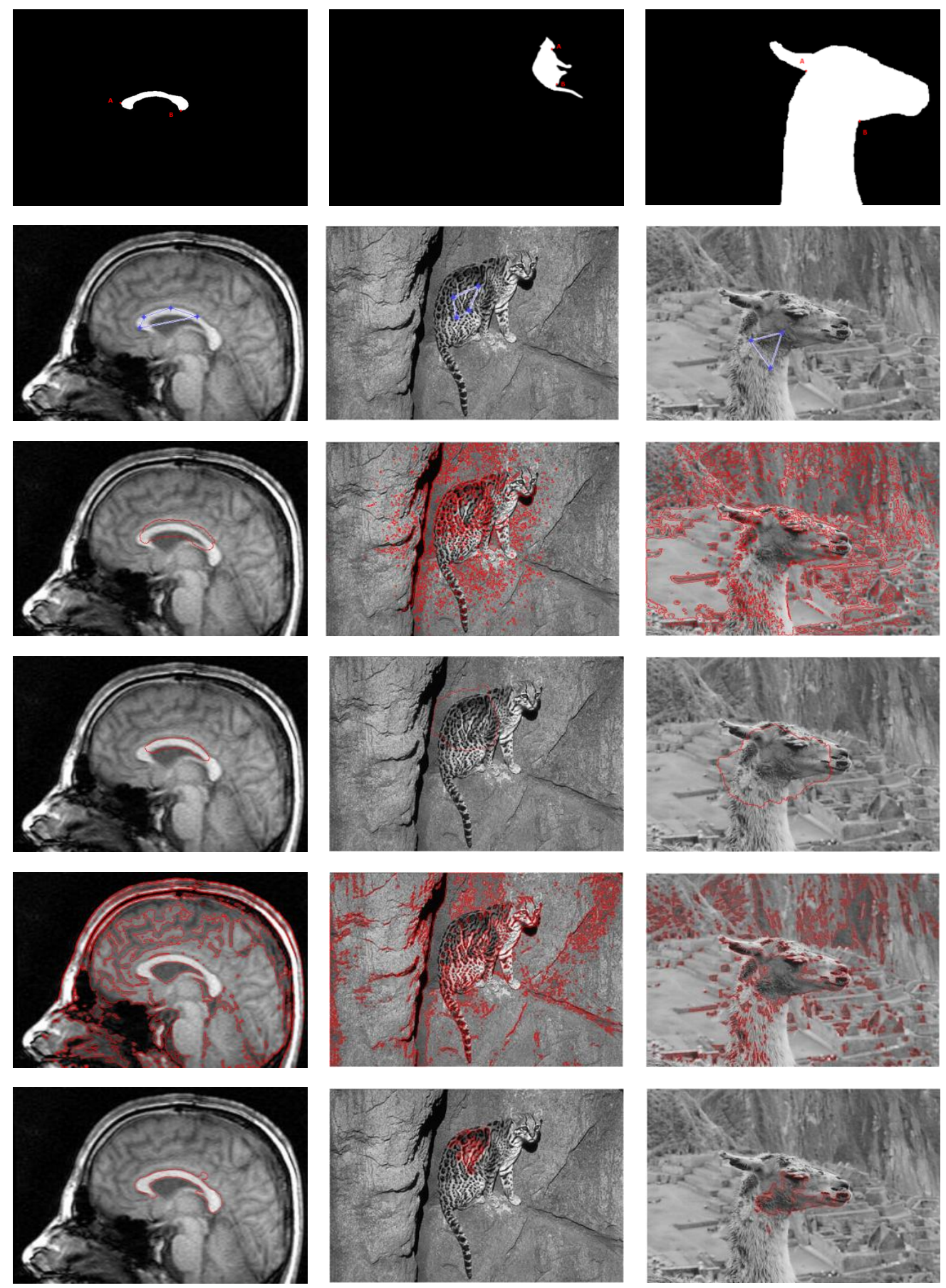

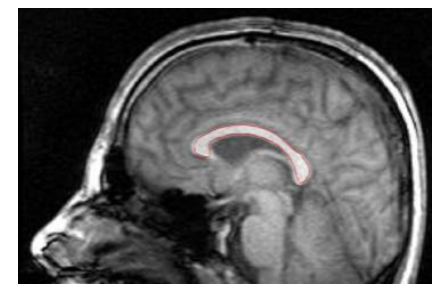

(a)

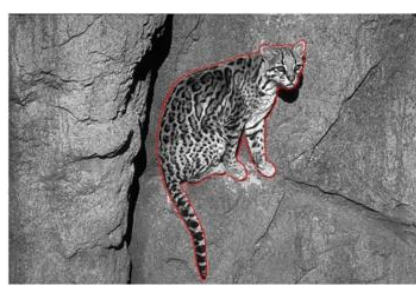

(b)

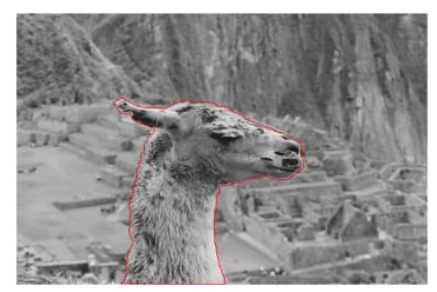

(c)

Fig. 5. Comparison of the experimental results. Row I: prior shapes, row II: initial contours, row III: results of the Chen's method, row IV: results of the Li's method, row V: results of AZ method, row VI: results of the CV's method and row VII: results of the proposed method. 
Table I. COMPARISON OFEXECUTIVE TIMES (S)

\begin{tabular}{lccccc}
\hline Methods & Our method & $\mathbf{C V}$ & $\mathbf{A Z}$ & Chen & $\mathbf{L i}$ \\
\hline Image (a) & $\mathbf{0 . 3 4 6 5}$ & 41.893 & 2.767 & 3.475 & 53.759 \\
Image (b) & $\mathbf{0 . 9 8 7 2}$ & 101.76 & 3.871 & 5.103 & 49.784 \\
Image (c) & $\mathbf{0 . 6 1 5 4}$ & 92.872 & 2.148 & 2.327 & 51.816 \\
\hline
\end{tabular}

Table II. $\quad$ LN'S INTER-REGION CONTRAST.

\begin{tabular}{lccccc}
\hline Methods & Our method & $\mathbf{C V}$ & $\mathbf{A Z}$ & Chen & $\mathbf{L i}$ \\
\hline Image (a) & $\mathbf{0 . 2 5 0 9}$ & 0.1711 & 0.0989 & 0.532 & 0.564 \\
Image (b) & $\mathbf{0 . 2 8 6 7}$ & 0.1254 & 0.0712 & 0.788 & 0.614 \\
Image (c) & $\mathbf{0 . 2 7 8 2}$ & 0.1103 & 0.0989 & 0.477 & 0.483 \\
\hline
\end{tabular}

Table III. $\quad$ LN'S INTRA-REGION CONTRAST.

\begin{tabular}{lccccc}
\hline Methods & Our method & $\mathbf{C V}$ & $\mathbf{A Z}$ & Chen & $\mathbf{L i}$ \\
\hline Image (a) & $\mathbf{1 . 9 6 e - 0 8}$ & $3.4 \mathrm{e}-07$ & 0.0262 & $6.4 \mathrm{e}-03$ & $9.3 \mathrm{e}-03$ \\
Image (b) & $\mathbf{1 . 7 2 e - 0 7}$ & $8.4 \mathrm{e}-07$ & 0.0435 & 0.0643 & 0.0242 \\
Image (c) & $\mathbf{3 . 4 4 e - 0 7}$ & $6.5 \mathrm{e}-07$ & 0.0911 & 0.0891 & 0.0176 \\
\hline
\end{tabular}
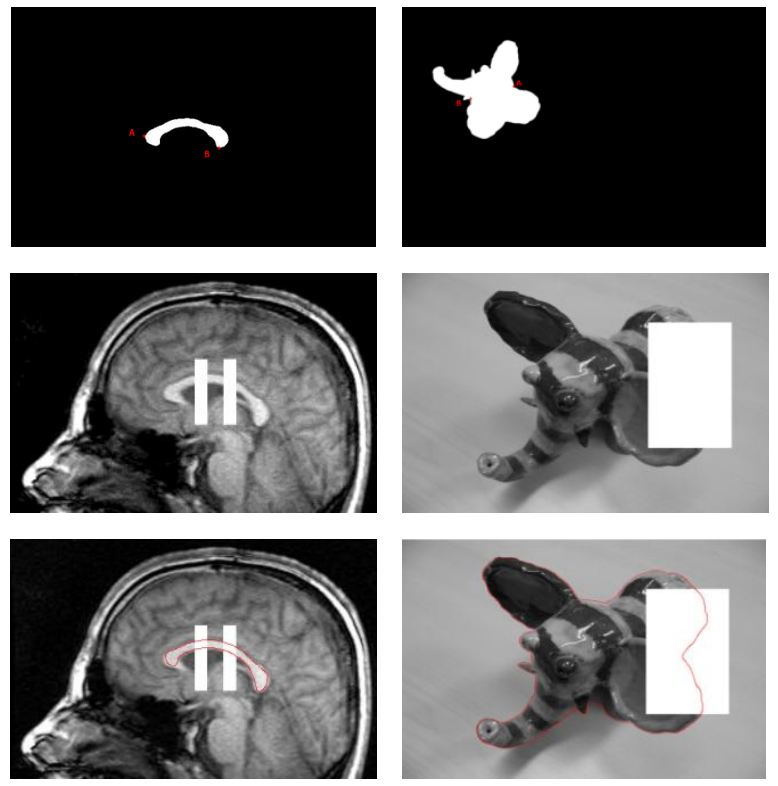

Fig. 6. Experimental results of the proposed method in presence of occlusion. Row I: prior shapes, row II: occluded images, and row III: segmentation results of the proposed method.

In Fig. 8 we demonstrate that the method we propose can be used even if the template is not available. As already specified, in this case we just need to fix $\phi_{r e f}=\phi$. The results obtained are subjectively promising.

Nevertheless, we should note that the effectiveness of our introduced model is limited in case of occlusion when the prior shape is incorrect. Furthermore, the model can lead to an inappropriate result if the recovery of the points $A$ and $B$ on the object to be segmented is inaccurate. This can limit the use of the method in automatic systems since it requires human interaction to be fully effective when used with prior shape. 


\section{Conclusion}

In this paper, we have presented a novel shape prior level set image segmentation algorithm based on the lattice Boltzmann model. It is effective in presence of noise, intensity inhomogeneities and partial occlusion, and can be used if the prior shape is not available by means of minor modification. Furthermore, it is local and thus highly parallelizable, which allows it to be a good candidate for parallel implementation. Experimental results using a graphics processing units on medical and real-world images have demonstrated the good performance of the proposed method in terms of efficiency, effectiveness and robustness.

Future work will be the development of an effective method for selecting the points $A$ and $B$ without human interaction. Furthermore, we will work on the extension of the proposed method to volume image segmentation and real-time objects (buildings, cars etc...) extraction in very high resolution satellite images.
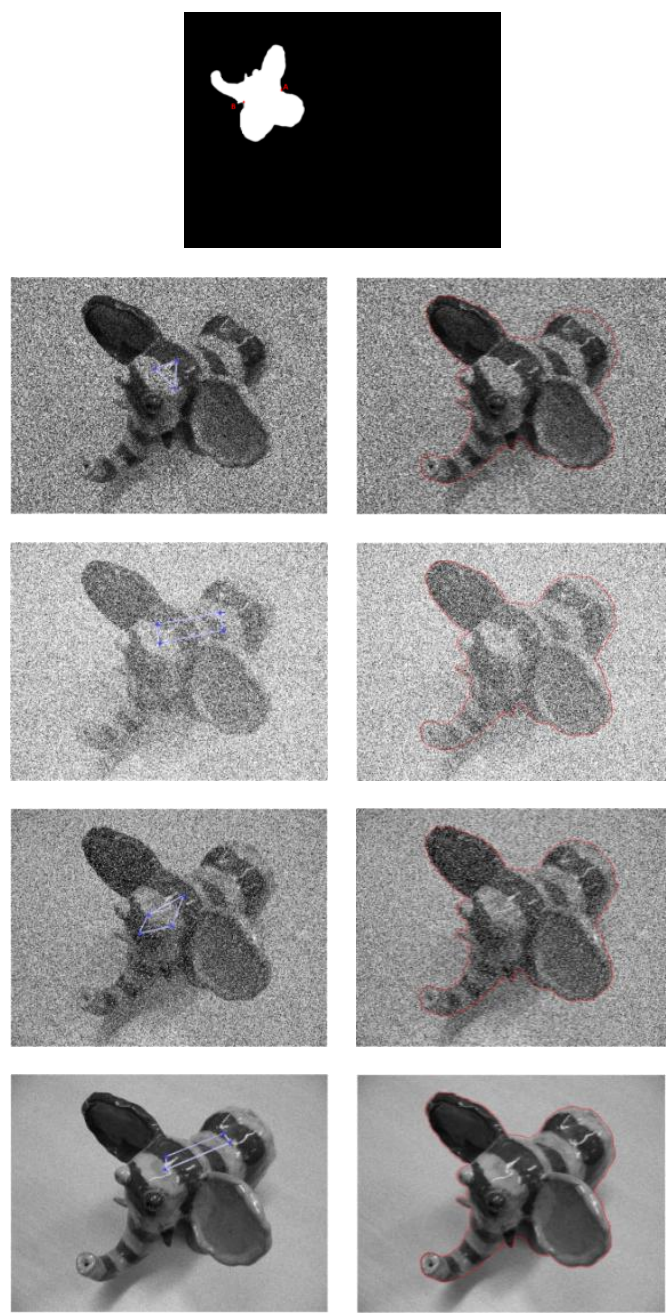

Fig. 7. Experimental results of the proposed method in presence of different types of noises. Row I: prior shapes; row II: image corrupted by the speckle noise; row III: image corrupted by gaussian white noise; row IV: image corrupted by the salt and pepper noise; row V: image corrupted by the poisson noise. Column I: initial contours, and column II: segmentation results of the proposed method. 

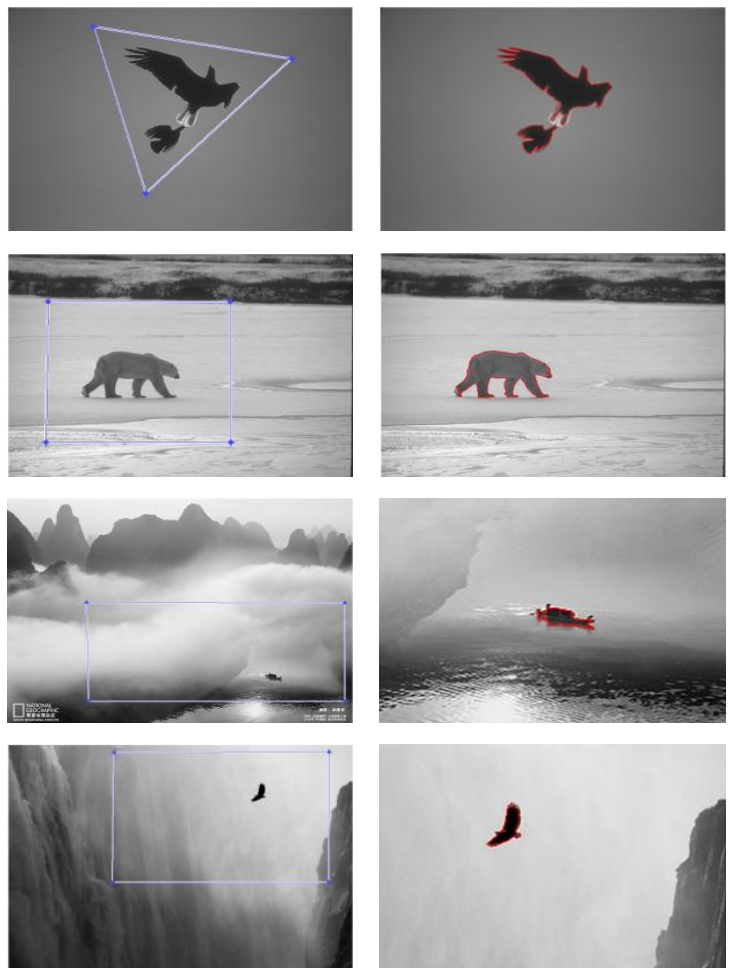

Fig. 8. Segmentation using the proposed method without prior shape. Column I: initial contours, and column II: segmentation results.

\section{REFERENCES}

[1] Y. Xu, J. Zhu, E. Chang and Z. Tu:Multiple clustered instance learning for histopathology cancer image classification, segmentation and clustering.IEEE Conference on Computer Vision and Pattern Recognition (CVPR), pp. 964-971 (2012)

[2] E. Kim, H. Li and X. Huang:A hierarchical image clustering cosegmentation framework.IEEE Conference on Computer Vision and Pattern Recognition (CVPR), pp. 686-693 (2012)

[3] M. Ben Salah, I. Ben Ayed and A. Mitiche: Active curve recovery of region boundary patterns. IEEE Transactions on Pattern Analysis and Machine Intelligence, 34(5):834-849(2012)

[4] K. Zhang, L. Zhang and S. Zhang:A variational multiphase level set approach to simultaneous segmentation and bias correction.IEEE 17th International Conference on Image Processing, pp. 4105-4108 (2010)

[5] S. Balla-Arabé, X.-B Gao and B. Wang: A fast and robust level set method for image segmentation using fuzzy clustering and lattice Boltzmann method. IEEE Transactions on Systems, Man and Cybernetics Part B: Cybernetics, 43(3):910-920(2013)

[6] C. Li, R. Huang, Z. Ding, J. Chris, D. N. Metaxas and J.C. Gore: A level set method for image segmentation in the presence of intensity inhomogeneities with application to MRI. IEEE Transactions on Image Processing, 20(7):2007-2016(2011)

[7] V.A. Prisacariu and I. Reid: Nonlinear shape manifolds as shape priors in level set segmentation and tracking.IEEE Conference on Computer Vision and Pattern Recognition (CVPR), pp. 2185-2192 (2011)

[8] S. Osher and J. Sethian. Fronts propagating and curvature dependent speed: algorithms based on Hamilton-Jacobi formulation. J. Comput. Phys., 79(1):12-49(1988)

[9] S. Balla-Arabé and X-B. Gao: Image multi-thresholding by combining the lattice Boltzmann model and a localized level set algorithm. Neurocomputing, vol. 93, pp. 106-114(2012) 
[10] M. Kass, A. Witkin and D. Terzopoulos: Snakes: Active contour models. International Journal of Computer Vision, $1(4): 321-331(1988)$

[11] X-B. Gao, B. Wang, D. Tao and X. Li: A relay level set method for automatic image segmentation. IEEE Transactions on Systems, Man and Cybernetics Part B: Cybernetics, 41(2):518-525(2011)

[12] R. Malladi, J. Sethian and B. Vemuri: A topology independent shape modeling scheme. Proceedings of SPIE Conference on Geometric Methods in Computer Vision II, vol. 2031, pp. 246-258(1993)

[13] V. Caselles, R. Kimmel and G. Sapiro: On geodesic active contours. International Journal of Computer Vision, 22(1):61-79(1997)

[14] N. Paragios and R. Deriche: Geodesic active contours for supervised texture segmentation.IEEE Conference on Computer Vision and Pattern Recognition (CVPR), pp. I: 1034-1040(1999)

[15] T. Chan and L. Vese: Active contours without edges. IEEE transactions on Image Processing, 10(2):266-277(2001)

[16] D. Mumford and J. Shah: Optimal approximations by piecewise smooth functions and associated variational problems. Commun. Pure Appl. Math., 42(5):577-685(1989)

[17] S. Osher and R. Fedkiw: Level set methods and dynamic implicit surfaces. Springer (2003)

[18] S. Balla-Arabé, B. Wang and X-B. Gao: Level set region based image segmentation using lattice Boltzmann method. Proceedings of the Seventh International Conference on Computational Intelligence and Security, pp. 1159-1163, (2011)

[19] X. He and L. Luo. Lattice Boltzmann model for incompressible Navier-Stokes equation. J. Stat. Phys., 88(3-4):927-944(1997)

[20] Ye Zhao: Lattice Boltzmann based PDE solver on the GPU. The Visual Computer, Springer, 24(5):323-333(2007)

[21] Y. Chen, Z. Yan and Y. Chu: Cellular automata based level set method for image segmentation. IEEE International Conference on Complex Medical Engineering, pp. 23-27(2007)

[22] C. Li, C. Xu, C. Gui and M.Fox: Distance regularized level set evolution and its application to image segmentation. IEEE Transactions on Image Processing, 19(12):3243-3254(2010)

[23] A. Hagan and Y. Zhao: Parallel 3D image segmentation of large data set on a GPU cluster. G. Bebis et al. (Eds.): ISVC 2009, Part II, LNCS 5876, Springer-Verlag Berlin Heidelberg, pp. 960-969(2009)

[24] M.D. Levine and A.M. Nazif: Dynamic measurement of computer generated image segmentations. IEEE Transactions on Pattern Analysis and Machine Intelligence, 25(7):155-164(1985)

[25] Y. Boycov, O. Veksler, and R. Zabih: Fast Approximate Energy Minimization Via Graph Cuts. IEEE Transactions on Pattern Analysis and Machine Intelligence, vol. 23, no. 11, pp. 1222-1239(2001)

[26] J. Malik, S. Belongie, T. Leung, and J. Shi: Contour and Texture Analysis for Image Segmentation. International Journal of Computer Vision, 43(1):7-27(2001)

[27] S. Zhu, and A. Yuille: Region Competition: Unifying Snakes, Region Growing, and Bayes/MDL for Multiband Image Segmentation. IEEE Transactions on Pattern Analysis and Machine Intelligence, vol. 18, no. 9, pp. 884-900(1996)

[28] C. Brun, N. Leporé, X. Pennec, Y. Chou, A. Lee et al.: A Nonconservative Lagrangian Framework for Statistical Fluid Registration-SAFIRA. IEEE Transactions on Medical Imaging, vol. 30, no. 2, pp. 184-202(2011)

[29] A. Nakhmani, and A. Tannenbaum: Self-crossing Detection and Location for Parametric active contours. IEEE Transactions on Image Processing, vol. 21, no. 7, pp. 3150-3156(2012)

[30] G. Charpiat, O. Faugeras and R. Keriven: Shape Statistics for Image Segmentation with prior.IEEE Conference on Computer Vision and Pattern Recognition (CVPR), pp. 1-6(2007) 
[31] N. Paragios and M. Rousson: Shape Priors for Level Set Representation.IEEE European Conference on Computer Vision (ECCV), pp. II: 78-93(2002)

[32] M. Ben Salah, I. Ben Ayed, and A. Mitiche: Active Curve Recovery of Region Boundary Patterns. IEEE Transactions on Pattern Analysis and Machine Intelligence, vol. 34, no. 5, pp. 834-849(2012)

[33] C. Benedek, X. Descombes, and J. Zerubia, "Building Development Monitoring in Multitemporal Remotely Sensed Image Pairs with Stochastic Birth-Death Dynamics,” IEEE Transactions on Pattern Analysis and Machine Intelligence, vol. 34, no. 1, pp. 33-50(2012)

[34] P. Arbelaez, M. Maire, C. Fowlkes, and J. Malik: Contour Detection and Hierarchical Image Segmentation. IEEE Transactions on Pattern Analysis and Machine Intelligence, vol. 33, no. 5, pp. 898-916(2011)

[35] Y. Yang, S. Hallman, D. Ramanan and C. Fowlkes: Layered Object Models for Image Segmentation. IEEE Transactions on Pattern Analysis and Machine Intelligence, vol. 34, no. 9, pp. 1731-1743(2012) 